\title{
Alleviating spatial confounding for areal data problems by displacing the geographical centroids: Supplementary Material
}

Marcos Oliveira Prates*, Renato Martins Assunção ${ }^{\dagger}$ and Erica Casitlho Rodrigues ${ }^{\ddagger}$

\section{S1 Results for the Linear Simulation}

Table 1 presents the median and $2.5 \%$ and $97.5 \%$ percentiles of these estimates of the mean estimate of 1000 replications of each fitted model.

\begin{tabular}{|c|c|c|c|c|c|c|c|c|c|c|}
\hline \multirow[b]{3}{*}{$\tau_{e}, \tau_{\theta}$} & \multirow[b]{3}{*}{ Model } & \multicolumn{9}{|c|}{ Scenario } \\
\hline & & \multicolumn{3}{|c|}{ ICAR spatial- $X$} & \multicolumn{3}{|c|}{ RHZ } & \multicolumn{3}{|c|}{ ICAR non-spatial- $X$} \\
\hline & & $\beta_{0}$ & $\beta_{1}$ & $\beta_{2}$ & $\beta_{0}$ & $\beta_{1}$ & $\beta_{2}$ & $\beta_{0}$ & $\beta_{1}$ & $\beta_{2}$ \\
\hline True & & 2 & 1 & -1 & 2 & 1 & -1 & 2 & 1 & -1 \\
\hline \multirow{5}{*}{$0.2,1$} & SPOCK $(\mathrm{CA}$ & $0(1.69,2.31)$ & ) & $-1.01(-1.83$, & $0(1.69,2.31)$ & $(0.67$ & $-1.00(-1.3$ & $2.00(1.69,2.31)$ & $1.00(0.64,1.33)$ & $-1.00(-1$. \\
\hline & RHZ & $2.00(1.69,2.31)$ & $1.00(0.66,1$. & $-1.01(-1.84,-($ & $0(1.69,2.31)$ & $1.00(0.67,1.31)$ & $-1.00(-1.30,-0.67)$ & $2.00(1.69,2.31)$ & $1.00(0.64,1.33)$ & $-1.00(-1.3$ \\
\hline & $\mathrm{H}$ & $2.00(1.69,2.31)$ & $00(0.66,1$. & $-1.01(-1.84,-0.13)$ & $0(1.69,2.31)$ & $1.00(0.67,1.31)$ & $-1.00(-1.30,-0.67)$ & $2.00(1.69,2.31)$ & $1.00(0.64,1.33)$ & $-1.00(-1.30$ \\
\hline & ICAR & $2.00(1.69,2.31)$ & $1.00(0.66,1.31)$ & $-1.01(-1.83,-0.16)$ & $0(1.69,2.31)$ & $1.00(0.67,1.31)$ & $-1.00(-1.33,-0.59)$ & $2.00(1.69,2.31)$ & $1.00(0.65,1.33)$ & $-1.00(-1.30,-0.69)$ \\
\hline & LM & $2.00(1.69,2.31)$ & $1.00(0.66,1.31)$ & $-1.01(-1.84,-0.13)$ & $2.00(1.69,2.31)$ & $1.00(0.67,1.31)$ & $-1.00(-1.30,-0.67)$ & $2.00(1.69,2.31)$ & $1.00(0.64,1.33)$ & $-1.00(-1.30,-0.68)$ \\
\hline \multirow{5}{*}{1,1} & CK (CARB & $0(1.86,2$ & )$(0$ & -1 & $(1.86$ & $(0.85$ & $(-1.1$ & $(1.86$ & $(0.8$ & -1.0 \\
\hline & $\mathrm{RHZ}$ & $2.00(1.86,2.14)$ & $.00(0.83,1.15)$ & $-1.00(-1.80,-0.22)$ & $0(1.86,2.14)$ & $1.00(0.85,1.14)$ & $-1.00(-1.13,-0.85)$ & $2.00(1.86,2.14)$ & $1.00(0.83,1.16)$ & $-1.00(-1.16,-0.83)$ \\
\hline & & $2.00(1.86,2.14)$ & $1.00(0.83,1.15)$ & $-1.00(-1.80,-0.22)$ & $0(1.86,2.14)$ & $1.00(0.85,1.14)$ & $-1.00(-1.13,-0.85)$ & $2.00(1.86,2.14)$ & $1.00(0.83,1.16)$ & $-1.00(-1.16,-0.83)$ \\
\hline & ICAR & $2.00(1.86,2.14)$ & $1.00(0.83,1.15)$ & $-1.01(-1.65,-0.34)$ & $0(1.86,2.14)$ & $1.00(0.85,1.14)$ & $-1.00(-1.46,-$ & $2.00(1.86,2.14)$ & $1.00(0.83,1.15)$ & $-1.00(-1.14,-0.84)$ \\
\hline & LM & $2.00(1.86,2.14)$ & $1.00(0.83,1.15)$ & $-1.00(-1.80,-0.22)$ & $2.00(1.86,2.14)$ & $1.00(0.85,1.14)$ & $-1.00(-1.13,-0.85)$ & $2.00(1.86,2.14)$ & $1.00(0.83,1.16)$ & $-1.00(-1.16,-0.83)$ \\
\hline \multirow{6}{*}{$1,0.2$} & $\mathrm{~K}(\mathrm{R}-$ & $0(1.86,2.14)$ & $0(0.75,1.22$ & $-1.00(-2.77,0.71)$ & $0(1.86,2.14)$ & $1.00(0.85,1$. & $-1.00(-1.15$ & $2.00(1.86,2$. & $0.99(0.75,1.24)$ & $-0.99(-1$ \\
\hline & $(\mathrm{CA}$ & , 2.14) & 07 & $(-2.7$ & $(1.86,2.14)$ & $(0.85,1.14)$ & $(-1.16,-0.85)$ & $(1.86,2.14)$ & $0.99(0.75,1.24)$ & $-1.00(-1$ \\
\hline & RHZ & $2.00(1.86,2.14)$ & $1.00(0.74,1.22)$ & $-1.01(-2.76,0.73)$ & $2.00(1.86,2.14)$ & $1.00(0.85,1.14)$ & $-1.00(-1.13,-0.85)$ & $2.00(1.86,2.14)$ & $0.99(0.74,1.26)$ & $-0.99(-1.26,-0.72)$ \\
\hline & & $2.00(1.86,2.14)$ & $1.00(0.74,1.22)$ & $-1.01(-2.76,0.73)$ & $2.00(1.86,2.14)$ & $1.00(0.85,1.14)$ & $-1.00(-1.13,-0.85)$ & $2.00(1.86,2.14)$ & $0.99(0.74,1.25)$ & $-0.99(-1.26,-0.72)$ \\
\hline & ICAR & $2.00(1.86,2.14)$ & $1.00(0.78,1.20)$ & $-0.99(-2.29,0.30)$ & $2.00(1.86,2.14)$ & $0.99(0.80,1.20)$ & $(-2.1$ & $2.00(1.86,2.14)$ & $1.00(0.78,1.20)$ & $-0.99(-1.19,-0.80)$ \\
\hline & LM & $2.00(1.86,2.14)$ & $1.00(0.74,1.22)$ & $-1.01(-2.76,0.73)$ & $2.00(1.86,2.14)$ & $1.00(0.85,1.14)$ & $-1.00(-1.13,-0.85)$ & $2.00(1.86,2.14)$ & $0.99(0.74,1.25)$ & $-0.99(-1.26,-0.72)$ \\
\hline
\end{tabular}

Table 1: Median summary of the posterior mean estimate from 1000 replicates in the simulation setting. The brackets contain the $2.5 \%$ and $97.5 \%$ posterior percentiles of fixed effects estimates in 1000 replicates. 


\section{S2 Results for the Nonlinear Simulation}

Figure 1 show the box plots of the ratio $\hat{\boldsymbol{\beta}} / \boldsymbol{\beta}^{*}$ in each one of the 1000 simulations of the quadratic and cubic simulation scenarios.
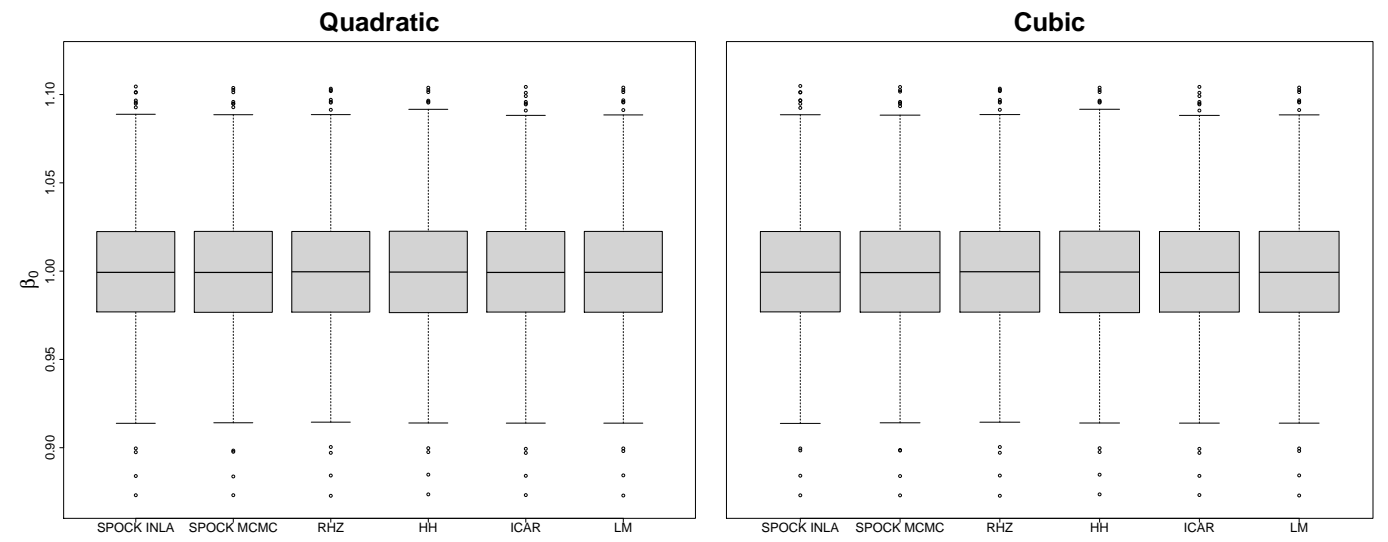

(a)
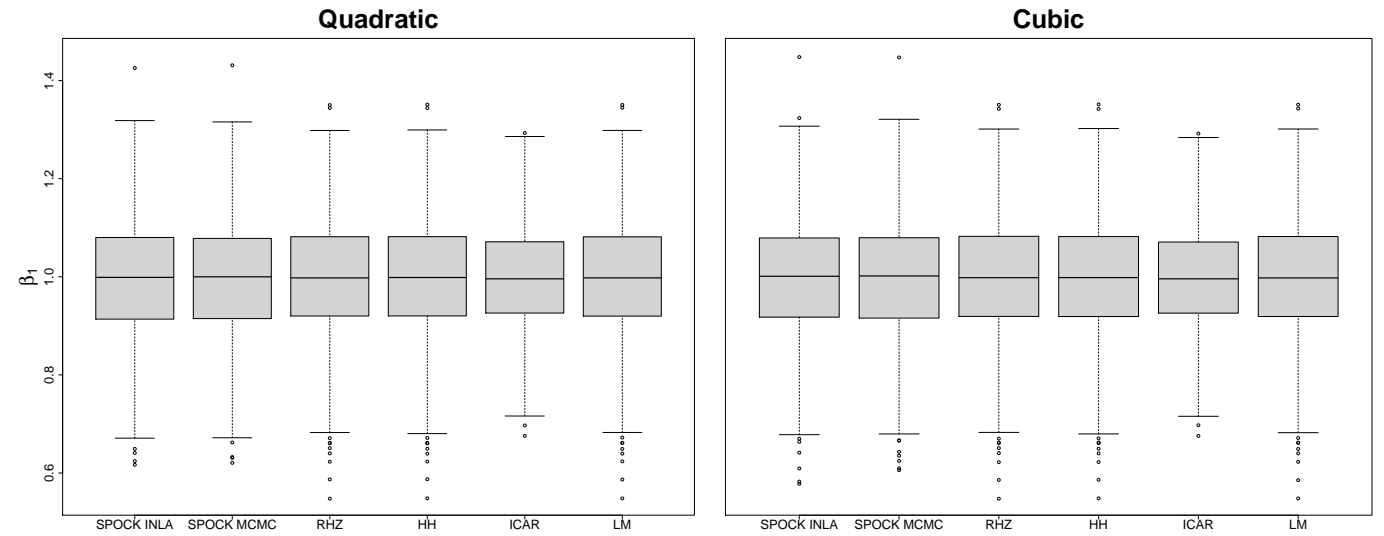

(b)
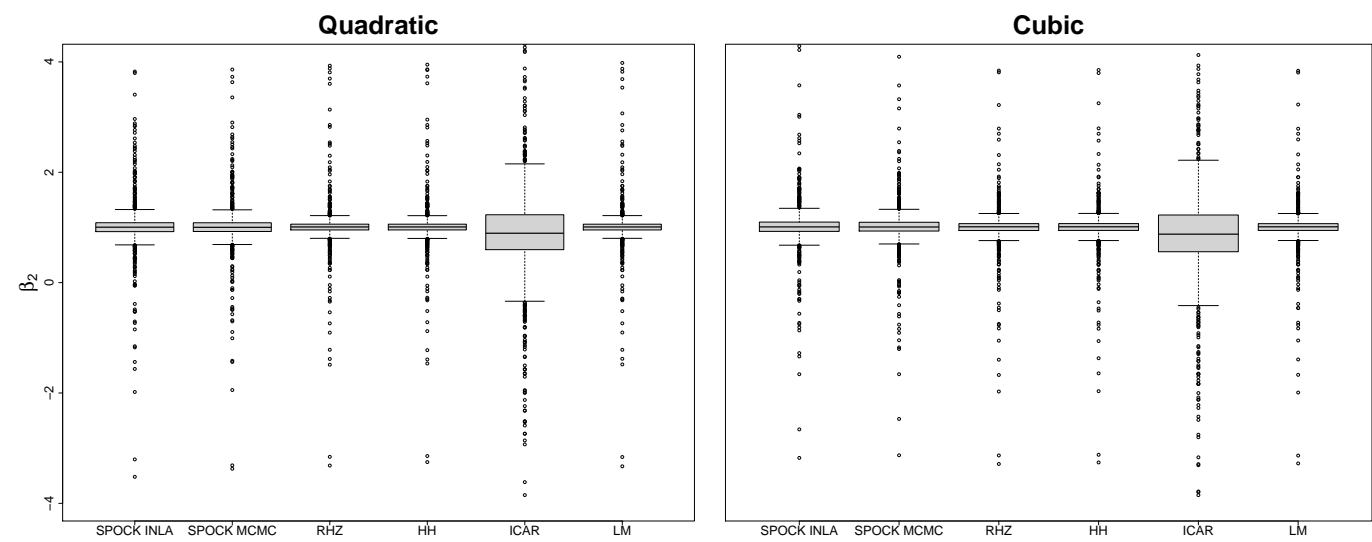

(c)

Figure 1: (a) Posterior estimates for $\beta_{0}$ under the quadratic and cubic dependencies. (b) Posterior estimates for $\beta_{1}$ under the different covariate dependencies. (c) Posterior estimates for $\beta_{2}$ under the covariate dependencies. 